\title{
The 1/e-strategy is sub-optimal for the problem of best choice under no information
}

\author{
F. Thomas Bruss ${ }^{\mathrm{a}, 1}$, L.C.G. Rogers ${ }^{\mathrm{b}, *}$ \\ ${ }^{\text {a } U n i v e r s i t e ́ ~ L i b r e ~ d e ~ B r u x e l l e s, ~ B e l g i u m ~}$ \\ ${ }^{\mathrm{b}}$ University of Cambridge, United Kingdom \\ Received 8 October 2020; received in revised form 15 April 2021; accepted 24 April 2021 \\ Available online $\mathrm{xxxx}$ \\ In Memory of Professor Larry Shepp
}

\begin{abstract}
This paper answers a long-standing open question concerning the $1 / e$-strategy for the problem of best choice. $N$ candidates for a job arrive at times independently uniformly distributed in $[0,1]$. The interviewer knows how each candidate ranks relative to all others seen so far, and must immediately appoint or reject each candidate as they arrive. The aim is to choose the best overall. The $1 / e$ strategy is to follow the rule: 'Do nothing until time $1 / e$, then appoint the first candidate thereafter who is best so far (if any).'

The question, first discussed with Larry Shepp in 1983, was to know whether the $1 / e$-strategy is optimal if one has 'no information about the total number of options'. Quite what this might mean is open to various interpretations, but we shall take the proportional-increment process formulation of Bruss and Yor (2012). Such processes are shown to have a very rigid structure, being time-changed pure birth processes, and this allows some precise distributional calculations, from which we deduce that the $1 / e$-strategy is in fact not optimal.

(C) 2021 Elsevier B.V. All rights reserved.
\end{abstract}

MSC: 60G40

Keywords: Optimal stopping; Secretary problem; Proportional increments; Pure birth process;

Hamilton-Jacobi-Bellman equation

* Correspondence to: Statistical Laboratory, University of Cambridge, Wilberforce Road, Cambridge CB3 oWB, United Kingdom.

E-mail addresses: tbruss@ulb.ac.be (F.T. Bruss), lcgr1@cam.ac.uk (L.C.G. Rogers).

1 Université Libre de Bruxelles, Département de Mathématique, CP 210, B-1050 Brussels, Belgium. 


\section{Dedication and background}

At the evening of Professor Larry Shepp's talk "Reflecting Brownian Motion" at Cornell University on July 11, 1983 (13th Conference on Stochastic Processes and Applications), Professor Shepp and Thomas Bruss ran into each other in front of the Ezra Cornell statue. Thomas was honoured to meet Prof. Shepp in person, but Larry replied "What are you working on?" And so Larry was the very first person with whom Thomas could discuss the 1/e-law of best choice resulting from the Unified Approach [2] which had been accepted for publication shortly before. Thomas was pleased to see the true interest Prof. Shepp showed for the 1/e-law. As many of us have seen before, when Larry was interested in a problem, elementary or not, then he was really deeply interested.

This article deals with an open question concerning the so-called $1 / e$-strategy for the problem of best choice, which is to wait and do nothing until time $1 / e$ and then to accept the first candidate (if any) who is best so far. The question which attracted our particular interest was whether this strategy is optimal if one has no initial information about the number $N$ of candidates. Bruss drew also attention to this open question in his own talk "The $e^{-1}$-law in best choice problems" at Cornell on July 14, 1983, and re-discussed it with Larry at several later occasions. A written record of somewhat related questions appeared on page 885 of Bruss [2] where he stated the conjecture that the $1 / e$-strategy is optimal in certain two-person games for a decision maker who faces an adversary trying to minimize the win probability. However, the two-person game situation is quite different from the open question discussed with Larry and will not be considered in this paper.

As far as we are aware, the last time the question discussed with Larry was addressed was in [7], and this may actually be the only written reference to the real open question. Bruss and Yor [7] studied another no-information stopping problem, the so-called last-arrival problem (l.a.p.). To prepare the paper's main result, they examined the hypothesis of no-information in a detailed way, and their conclusions will be used in the present paper. Bruss and Yor [7] also used these to give, as a side-result, an alternative proof of the $1 / e$-law. However, as they pointed out, their approach did not contribute new insights for the open question.

The present article proves that the $1 / e$-strategy is not optimal under the interpretation of "no information' used by Bruss and Yor [7]. It thus closes a 37 year gap. The many various forms of the optimal stopping problem have been a topic of recurring interest since the literature was started, and we do not attempt to survey the fascinating range of results and paradoxes in this area. A recent article by Gnedin [9] discusses the literature thoroughly and presents some surprising new results.

\section{The unified approach}

The so-called 1/e-law of best choice is a result obtained in the Unified Approach-model of Bruss [2]. The model is as follows:

Unified Approach: Suppose $N>0$ points are IID $U[0,1]$. Points are marked with qualities which are supposed to be uniquely rankable from 1 (best) to $N$ (worst). The goal is to maximize the probability of stopping online, and without recall on a preceding observation, on rank 1 .

This model was suggested for the best choice problem (secretary problem) for an unknown number $N$ of candidates. (More general payoff-functions for the same model were studied in [5].) 
Now, if we contemplate the probability of picking the best candidate, we immediately face the question 'What is $N$ ?' If $N$ is fixed and known, this is just the classical secretary problem. But if we take a Bayesian point of view and suppose a prior distribution for $N$, with arrivals coming at times $t \in \mathbb{N}$, Abdel-Hamid et al. [1] showed that the problem may not only lead to so-called stopping islands [11], but, much worse, that for any $\epsilon>0$ there exists a sufficiently unfavourable distribution $\{P(N=n)\}_{n=1,2, \ldots}$ to reduce the value of the optimal to less than $\epsilon$. In other words, if $N$ is allowed an arbitrary prior, optimality may mean almost nothing. These discouraging facts prompted efforts to find more tractable models, such as the model of Stewart [13], and the one of Cowan and Zabczyk [8] and its generalization studied in [3].

The philosophy behind the unified approach of Bruss [2] was different. The approach was to suppose that arrival times are in $[0,1]$ and to study so-called $x$-strategies, where you do nothing until time $x$, and thereafter pick the first record. One of the main results of that paper was that the $1 / e$-strategy gives a success probability of at least $1 / e$ whatever the prior distribution of $N$, and that no other $x$-strategy does this well. This robustness suggests that the $1 / e$-strategy is somehow special, and the open question became natural.

It is relevant to mention here that a similar phenomenon of robustness shows up in different forms. One is what Bruss and Samuels [6] called 'quasi-stationarity', meaning essentially that the optimal strategy may (even for rather general payoffs) hardly depend on the number of candidates observed so far. More remarkably, for so-called Pascal processes, optimal strategies do not depend at all on the number of preceding observations (For their characterization see [4]).

\subsection{The open question}

First we need to be clear about what exactly we mean by optimality of a strategy under no information on $N$. We see objects arriving at the times of a counting process $\left(N_{t}\right)_{0 \leq t \leq 1}$, $N_{0}=0$. When an object arrives, we see its rank relative to all objects that have arrived so far. Defining

$$
\begin{aligned}
\rho_{t}^{\prime} & =\text { relative rank of object arriving at } t \text { if } \Delta N_{t}=1 \\
& =0 \quad \text { otherwise, }
\end{aligned}
$$

we define $\mathcal{F}_{t}=\sigma\left(N_{u}, \rho_{u}^{\prime} ; u \leq t\right)$. The law of $\left(N_{t}\right)_{0 \leq t \leq 1}$ is $P_{\theta}$ for some $\theta \in \Theta$, where $\left\{P_{\theta}: \theta \in \Theta\right\}$ is the collection of possible laws considered.

The notion that 'we have no prior information at all on $N$ ' means that we are only going to consider strategies which are $\left(\mathcal{F}_{t}\right)$-stopping times. That is, the strategies allowed can only know the arrival times (and relative ranks) of the individuals, not the value of $\theta \in \Theta$. This is the viewpoint of classical statistics.

To understand the sense of optimality, define the process $\rho$ by

$$
\begin{aligned}
\rho_{t} & =\text { overall rank of object arriving at } t \text { if } \Delta N_{t}=1 \\
& =0 \quad \text { otherwise. }
\end{aligned}
$$

Let $\mathcal{T}$ denote the set of all $\left(\mathcal{F}_{t}\right)$-stopping times. Then the value of using $\tau \in \mathcal{T}$ is

$$
R(\theta, \tau)=P_{\theta}\left[\rho_{\tau}=1\right]
$$

We denote by $\tau^{*}$ the stopping time corresponding to the $1 / e$ strategy, which is simply $\tau^{*}=$ $\inf \left\{t \geq 1 / e: \rho_{t}^{\prime}=1\right\}$, where it is understood that $\tau^{*}=1$ if no such $t$ exists, and that, in this case, we lose by definition. In these terms, the open question is stated precisely as follows: 
True or false:

$$
\forall \theta \in \Theta, \forall \tau \in \mathcal{T}, \quad R\left(\theta, \tau^{*}\right) \geq R(\theta, \tau) ?
$$

Of course, the set $\Theta$ of possible laws of $\left(N_{t}\right)_{0 \leq t \leq 1}$ plays an important rôle in the conjecture. For example, if $\Theta$ contained just one law, under which $\left(N_{t}\right)_{0 \leq t \leq 1}$ was the counting process of ten $U[0,1]$ arrival times, then clearly the $1 / e$ strategy would not be optimal in the sense of (2). We shall shortly explain exactly what set of laws is considered here.

\subsection{A related problem}

We return to the related last-arrival problem under no information (l.a.p.) studied in [7]. In this model an unknown number $N$ of points are IID $U[0,1]$ random variables, and an observer, inspecting the interval $[0,1]$ sequentially from left to right, wants to maximize the probability of stopping online on the very last point. No information about $N$ whatsoever is given. Only one stop is allowed, and this again without recall on preceding observations.

Central to the approach of Bruss and Yor [7] is the choice of the family $\Theta$ of laws of the counting process $\left(N_{t}\right)_{0 \leq t \leq 1}$. These authors present arguments (based on the properties of IID $U[0,1]$ arrival times) to justify their focus on the family of what they call proportionalincrements (p.i.) counting processes. We shall not repeat all the reasoning which leads to this choice of counting processes, but we show its basic motivation and explain why we take its implications as our starting point.

Bruss and Yor [7] defined a p.i.-process as follows: A stochastic process $\left(N_{t}\right)$ defined on a filtered probability $\operatorname{space}\left(\Omega, \mathcal{F},\left(\mathcal{F}_{t}\right), P\right)$ with natural filtration $\mathcal{F}_{t}=\sigma\left\{N_{u}: u \leq 1\right\}$ is a p.i.-counting process on $] 0, \infty[$, if

$$
\begin{aligned}
& \forall t \text {, conditional on } N_{t}>0, \forall s \geq 0, \\
& \mathrm{E}\left(N_{t+s}-N_{t} \mid \mathcal{F}_{t}\right)=\frac{s}{t} N_{t} \text { a.s. }
\end{aligned}
$$

The meaning of proportional is well understood in this definition. Moreover, three out of the four conclusions 1.-4. in [7], implying this definition, are proved to be compelling for combining the IID $U[0,1]$ - hypothesis for arrival times with the hypothesis that no prior information on $N$ can be used. Only Conclusion 3 (on page 3244) makes a concession. Here these authors use an (unprovable) tractability argument to justify why an unknown random variable, of which the expectation must be zero, is put equal to zero.

Why a concession? It is important to note that, if one has no information on $N$, then the time of the first arrival $T_{1}$ is a particularly delicate point. It is the smallest order statistic of all arrival $N$ times. However, it is exactly this one which escapes any distributional prescription because the no-information setting does not allow us to assume a prior distribution $\{P(N=n)\}_{n=1,2, \ldots}$. Hence, if one wants to confine one's interest to a well-posed problem, as Bruss and Yor [7] did, one has to make a concession somewhere if one wants to properly define a relevant decision process in the no-information case. The mentioned concession seemed the least restrictive and almost compelling, but, more importantly, Bruss and Yor [7] found a solid a-posteriori justification for their tractability argument. The solution of the l.a.p. they obtained for p.i.processes satisfied the criteria of Hadamard [10] for the solution of a well-posed problem. Bruss and Yor [7] found these criteria convincing.

Now note that the only difference between the l.a.p. and our open problem (how to find rank 1 ) is that we want to stop on the last record of the arrival process, and not on the last point. By 
the IID-hypothesis for arrival times of absolute ranks, Rényi's Theorem of relative ranks [12] implies that the $k$ th point is a record with probability $1 / k$ independently of preceding arrivals. Thus the basic arrival process $\left(N_{t}\right)$ is not affected and can be chosen exactly the same! This is why, confining our interest to well-defined problems only, we suppose that $\left(N_{t}\right)$ is a p.i.-process in the sense of Bruss and Yor [7], from which we take the following definition.

Definition 2.1. A p.i.- counting process is a counting process whose compensator is $\lambda_{t} \equiv N_{t} / t$, so that $(t \in(0,1])$

$$
M_{t} \equiv N_{t \vee T_{1}}-N_{T_{1}}-\int_{T_{1}}^{t \vee T_{1}} \frac{N_{s}}{s} d s \quad \text { is a martingale in its own filtration, }
$$

where $T_{1} \equiv \inf \left\{t: N_{t}=1\right\}$ is the first jump time of the counting process.

The class $\Theta$ of counting processes will be the class of all p.i.-processes, and the meaning of all the notation appearing in the statement (2) has now been defined.

\section{Analysis of the open question}

Our analysis starts with the following little result, whose proof is immediate from the statement.

Proposition 3.1. Suppose that $(N)$ is a p.i.-counting process. If we define $\tilde{N}(u)=N\left(e^{u}\right)$ for $u \in(-\infty, 0]$, and $t_{1}=\log T_{1}$, then

$$
\begin{aligned}
M\left(e^{u}\right) & =N\left(e^{u} \vee T_{1}\right)-N\left(T_{1}\right)-\int_{T_{1}}^{e^{u} \vee T_{1}} \frac{N_{s}}{s} d s \\
& =\tilde{N}\left(u \vee t_{1}\right)-\tilde{N}\left(t_{1}\right)-\int_{t_{1}}^{u \vee t_{1}} \tilde{N}(s) d s
\end{aligned}
$$

is a martingale in its own filtration, so $(\tilde{N})$ is a pure birth process, started with one individual at time $t_{1}$.

So the requirement that $\left(N_{t}\right)$ be a p.i.- counting process is not in fact very general - apart from the choice of the time $(\tilde{N})$ starts, the behaviour is uniquely determined!

If we run a pure birth process from $\tilde{N}_{u}=1(u<0)$ to time 0 , the PGF of $\tilde{N}_{0}$ is easily shown to be

$$
E\left[z^{\tilde{N}_{0}} \mid \tilde{N}_{u}=1\right]=\frac{z e^{u}}{1-z\left(1-e^{u}\right)} \quad(z \in[0,1]),
$$

so that $\tilde{N}_{0}$ is $1+$ geometric $\left(e^{u}\right)$. Obviously, from (4) we deduce

$$
E\left[z^{\tilde{N}_{0}} \mid \tilde{N}_{u}=k\right]=\left\{\frac{z e^{u}}{1-z\left(1-e^{u}\right)}\right\}^{k} \quad(z \in[0,1]),
$$

Thus if we see a record in the process $(\tilde{N})$ at time $u<0$, at the arrival of the $n$th observation, the probability that this is the best overall will be

$$
\tilde{\pi}_{n}(u) \equiv E\left[\frac{n}{\tilde{N}_{0}} \mid \tilde{N}_{u}=n\right]=n \int_{0}^{1} \frac{d z}{z}\left\{\frac{z e^{u}}{1-z\left(1-e^{u}\right)}\right\}^{n} .
$$


In terms of the original process $(N)$, if we see a record at the arrival of the $n$th observation at time $t \in(0,1)$, then the probability that this is the best overall is

$$
\pi_{n}(t) \equiv E\left[\frac{n}{N_{1}} \mid N_{t}=n\right]=n \int_{0}^{1} \frac{d z}{z}\left\{\frac{z t}{1-z(1-t)}\right\}^{n} .
$$

Clearly this has to be increasing in $t$, but from numerics it appears also to be decreasing in $n$. We can prove that this has to be the case, as follows. If we fix $t \in(0,1)$ then conditional on $N_{t}=n$ we have that

$$
\frac{N_{1}}{n}=\xi_{n} \equiv \frac{n+W_{1}+\cdots+W_{n}}{n},
$$

where the $W_{j}$ are IID geometrics. Now $\left(\xi_{n}\right)$ is a reversed martingale in the exchangeable filtration, so $\left(1 / \xi_{n}\right)$ is a reversed submartingale in the exchangeable filtration, so its expectation decreases with $n$.

\section{The value of a fixed threshold rule}

Suppose we use a fixed threshold rule, that is, we do nothing until $u \geq b$ and then we take the first record thereafter. The $1 / e$ rule corresponds to the special case $b=-1$. What is the value of this?

If $\tilde{N}_{b}=n$, then the distribution of the number $Y$ of further observations is known, and is a negative binomial distribution:

$$
P[Y=y]=q^{y} p^{n}\left(\begin{array}{c}
n+y-1 \\
y
\end{array}\right) \quad(y \geq 0),
$$

where $p=\exp (b)$. Given that $Y=y$, the probability that the best comes after the first $n$ observations is $y /(n+y)$, and the probability that the first record after $u=b$ is actually the best is

$$
P[\text { first record after } n \text { is best|best comes after first } n, Y=y]=\frac{1}{y} \sum_{j=1}^{y} \frac{n}{n+j-1} .
$$

Thus we have an expression for the probability that we pick the best using this rule:

$$
P[\text { win }]=\sum_{y \geq 1} P[Y=y] \frac{n}{n+y} \sum_{j=1}^{y} \frac{1}{n+j-1} .
$$

\subsection{The special case $n=1$}

Let us firstly observe that for $t \in(0,1)$

$$
f_{j}(t) \equiv \sum_{k \geq j} \frac{t^{k}}{k}=\int_{0}^{t} \frac{s^{j-1}}{1-s} d s
$$

from which we see that $f_{1}(t)=-\log (1-t)$. In the special case $n=1$, we have

$$
\begin{aligned}
P \text { [win] } & =\sum_{k \geq 1} q^{k} p \frac{1}{1+k} \sum_{j=1}^{k} \frac{1}{j} \\
& =p q^{-1} \sum_{j \geq 1} \frac{1}{j} f_{j+1}(q)
\end{aligned}
$$




$$
\begin{aligned}
& =p q^{-1} \int_{0}^{q} \sum_{j \geq 1} \frac{s^{j}}{j} \frac{d s}{1-s} \\
& =p q^{-1} \int_{0}^{q}\left(\int_{0}^{s} \frac{d v}{1-v}\right) \frac{d s}{1-s} \\
& =\frac{1}{2} p q^{-1}\left(\int_{0}^{q} \frac{d v}{1-v}\right)^{2} \\
& =\frac{1}{2} p q^{-1}(\log (1-q))^{2} .
\end{aligned}
$$

Similarly, from (6) we have $\left(p \equiv 1-q \equiv e^{u}\right)$

$$
\begin{aligned}
\tilde{\pi}_{1}(u) & =\sum_{k \geq 0} \frac{q^{k} p}{1+k} \\
& =\frac{p}{q} f_{1}(q) \\
& =-\frac{p}{q} \log (1-q) .
\end{aligned}
$$

\section{The Hamilton-Jacobi-Bellman equations}

If $V_{n}(u)$ denotes the value of being at time $u \leq 0$ with $n$ events already observed, none of them at time $u$, then the HJB equations of optimal control for the $V_{n}$ are

$$
\begin{aligned}
0 & =\dot{V}_{n}(u)+n\left\{\frac{n}{n+1} V_{n+1}(u)+\frac{1}{n+1} \max \left\{V_{n+1}(u), \tilde{\pi}_{n+1}(u)\right\}-V_{n}(u)\right\} \\
& =\dot{V}_{n}+n\left(V_{n+1}-V_{n}\right)+\frac{n}{n+1}\left(\tilde{\pi}_{n+1}-V_{n+1}\right)^{+},
\end{aligned}
$$

together with the boundary conditions $V_{n}(0)=0$. The solution is then the value function $V_{n}(u)$.

Now, if the answer to the open question is affirmative, then for all $n, V_{n}=V_{n}^{*}$, where $V^{*}$ is the value function for using the $1 / e$ strategy, which we actually know reasonably explicitly; it is given by the right-hand side of (11), where the dependence on the time $u<0$ comes via the parameter $p=e^{u}$ of the negative binomial distribution (9). Now by inspection of the HJB equations, we see that the optimal rule will be that we stop when we see a new record at time $u<0$ when there are $N_{u}=n$ observations in total if and only if

$$
\tilde{\pi}_{n}(u)>V_{n}(u) .
$$

If the 1/e-strategy is optimal, this would say that

$$
\tilde{\pi}_{n}(u)>V_{n}^{*}(u) \quad \text { if and only if } u>-1 \text {. }
$$

We can investigate this numerically by calculating $\tilde{\pi}_{n}(-1)$ and $V_{n}^{*}(-1)$ and comparing them; the results are plotted in Fig. 1.

We see that $\tilde{\pi}_{n}(-1)>V_{n}^{*}(-1)$ for all $n$, and that the gap narrows as $n$ increases. Hence the answer to the open question is No. The $1 / e$-strategy is not optimal. 


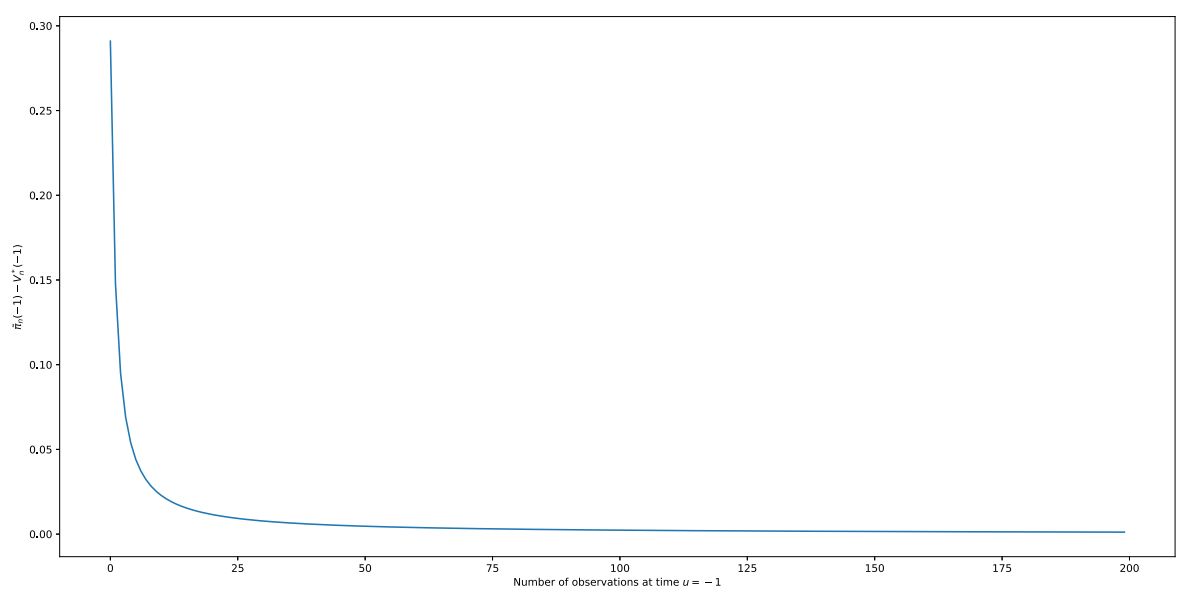

Fig. 1. $\tilde{\pi}_{n}(-1)-V_{n}^{*}(-1)$.

\subsection{Analytic proof}

It is nice to see without resorting to numerics that the answer must be No, by considering the special case $n=1$. From (13) and (14) we see that for the $1 / e$ rule where $p=e^{-1}, u=-1$

$$
\tilde{\pi}_{1}(u)-V_{1}^{*}(u)=\frac{p}{2 q}\left[-2 \log (1-q)-(\log (1-q))^{2}\right]=p / 2 q
$$

which is clearly positive.

\subsection{Should this have been obvious?}

A closer look at (18) may attract our attention; we may be surprised how big the difference between $\tilde{\pi}_{1}(u)$ and the performance of the $1 / e$-strategy can actually become, namely the first one is twice the second one for $u=-1$ (i.e. for $t=1 / e$ in $[0,1]$-time.) Is it then not surprising that the non-optimality of the $1 / e$-strategy did not follow already from simpler comparisons?

No, not easily. First note that we compare here a conditional win probability, given that we have a (granted) record at time $T_{1} \leq 1 / e$, with the unconditional win probability of the $1 / \mathrm{e}$ strategy. Fortunately, this was all that was needed to show that the $1 / e$-strategy is not optimal, namely showing that there are situations where it is definitely strictly sub-optimal to pass over the first arrival, even when arriving at some time $1 / e-\epsilon$ with $\epsilon>0$.

This however does, a priori, not say much about the absolute win probability of the $1 / e$ strategy! To see this, suppose that for some small $\epsilon>$ we have $T_{1} \in[1 / e-\epsilon, 1 / e+\epsilon]$, and that $N$ is not large. The latter is quite probable if $T_{1}$ is close to $1 / e$. Then $T_{1}$ will be almost equiprobable in the left or right half of $[1 / e-\epsilon, 1 / e+\epsilon]$ for $\epsilon$ sufficiently small. If it is in the right half, however, the $1 / e$-strategy will accept it all the same, but now with a strictly greater win probability since $\tilde{\pi}_{1}(t)$ is strictly increasing in $t$.

A second reason why non-optimality is not that evident lies in the interplay of time and the number of arrivals (see (6) and (8)). If we have (by simpler estimates) no sufficient incentive to accept at time $t<1 / e$ a record, being the $n$th arrival, say, we have even less incentive if it was the $(n+1)$ th arrival. With each additional arrival before time $1 / e$ increases the expected 
number of arrivals thereafter, and so does the expected number of those arriving after time $1 / e$. This increases the incentive to wait, but then we also have to wait for a record! This may bring us quickly behind time $1 / e$. If we get there a record, then, as said in the first scenario, so much the better.

These two scenarios exemplify how important it is to have precise answers, and not only estimates, even if one has reasonably good ones. It is the preceding approach which offered these precise answers, and it made things definite: In the no-information case, the $1 / e$-strategy is not optimal.

\section{Declaration of competing interest}

The authors declare that they have no known competing financial interests or personal relationships that could have appeared to influence the work reported in this paper.

\section{Acknowledgements}

The authors would like to thank very warmly Professor Philip Ernst for his precious indirect and direct contributions to this article. When Philip organized in 2018 the most memorable conference at Rice University in honour of Larry Shepp, he motivated many of us to look at harder problems. And so this old open problem turned up again and attracted attention. Philip's interest in this problem and his numerous interesting comments on, and discussions of, a former version of this paper, were very encouraging.

\section{References}

[1] A.R. Abdel-Hamid, J.A. Bather, G.B. Trustrum, The secretary problem with an unknown number of candidates, J. Appl. Probab. 19 (1982) 619-630.

[2] F.T. Bruss, A unified approach to a class of best choice problems with an unknown number of options, Ann. Probab. 12 (1984) 882-889.

[3] F.T. Bruss, Invariant record processes and applications to best choice modelling, Stochastic Process. Appl. 30 (2) (1988) 303-316.

[4] F.T. Bruss, L.C.G. Rogers, Pascal processes and their characterization, Stochastic Process. Appl. 37 (2) (1991) $331-338$.

[5] F.T. Bruss, S.M. Samuels, A unified approach to a class of optimal selection problems with an unknown number of options, Ann. Probab. 18 (1987) 824-830.

[6] F.T. Bruss, S.M. Samuels, Conditions for quasi-stationarity of the Bayes rule in selection problems with an unknown number of rankable options, Ann. Probab. (1990) 877-886.

[7] F.T. Bruss, M. Yor, Stochastic processes with proportional increments and the last-arrival problem, Stochastic Process. Appl. 122 (9) (2012) 3239-3261.

[8] R. Cowan, J. Zabczyk, An optimal selection problem associated with the Poisson process, Theory Probab. Appl. 23 (3) (1979) 584-592.

[9] A. Gnedin, How to beat the 1/e-strategy of best choice (the random arrivals problem), 2021, arXiv preprint arXiv:2102.10672.

[10] J. Hadamard, Sur les problèmes aux dérivées partielles et leur signification physique, Princeton University Bulletin, 1902, pp. 49-52.

[11] E.L. Presman, I.M. Sonin, The best choice problem for a random number of objects, Theory Probab. Appl. 17 (4) (1973) 657-668.

[12] A. Rényi, Théorie des éléments saillants d'une suite d'observations, Ann. Sci. Univ. Clermont. Math. 8 (2) (1962) 7-13.

[13] T.J. Stewart, The secretary problem with an unknown number of options, Oper. Res. 29 (1) (1981) $130-145$. 